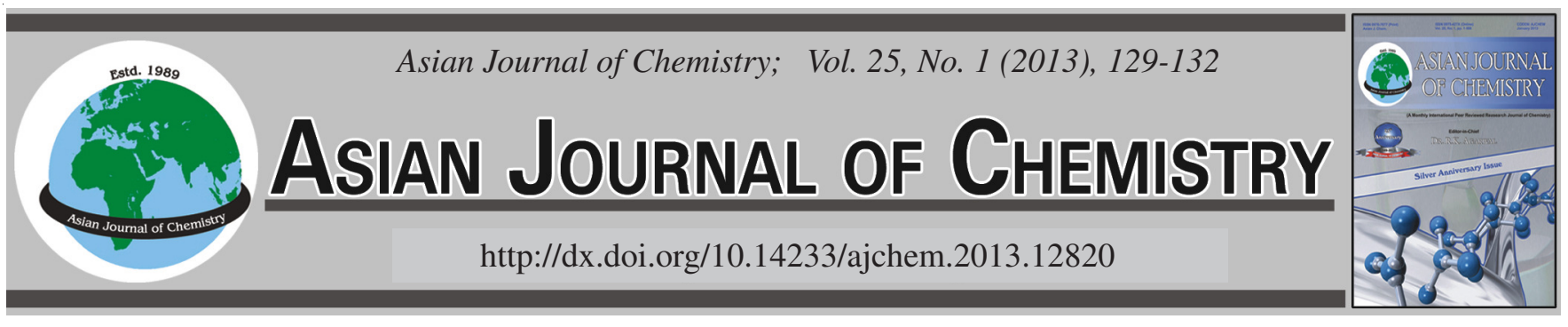

\title{
Rheological Behaviour of Magneto Thixotropic Gel
}

\author{
Hongzhan Lv $v^{1, *}$ XILIANg Chen ${ }^{1}$ and Xichang Liang ${ }^{2}$
}

\begin{abstract}
${ }^{1}$ Ningbo Institute of Material Technology and Engineering, Chinese Academy of Sciences, Ningbo 315201, P.R. China
${ }^{2}$ The State Key Laboratory of Mechanical Transmissions, Chongqing University, Chongqing 400044, P.R. China

*Corresponding author: Fax: +86 574 86688137; Tel: +86 574 86688131; E-mail: hongzhanlv@ 126.com
\end{abstract}

\begin{abstract}
A new type of smart material namely magneto-thixotropic gel is proposed in this study. To investigate its rheological behaviour, an experiment is designed to predict the interaction among the shear strain rate, shear stress, magnetic induction and viscosity. By theoretical analysis and interactive processing on the testing data, compared with magneto-rheological fluids, the experimental results indicate that the magneto-thixotropic gel possesses better linear magnetization property and higher shear yield stress, with the shear strain rate changes, the shear stress keeps relatively stable trend when exposed to certain magnetic field, meanwhile, the computational viscosity will rise with the decrease of the shear strain rate and increasing of magnetic induction and keeps linearly increase with the increasing of magnetic induction under the same shear strain rate.
\end{abstract}

Key Words: Magneto-thixotropic gel, Rheological behaviour, Magnetization, Power transmission, Stability.

\section{INTRODUCTION}

Magneto-thixotropic gel, as a new type of smart material, whose viscosity can change from colloidal state to semi-solid or solid state within milliseconds when exposed to a magnetic field, compared with magneto-rheological fluids. The magnetothixotropic gel has better anti-sedimentation ability and antiagglomeration ability ${ }^{1}$, therefore, the magneto-thixotropic gel has been developed for industrial and military use and could be used in such field as vehicles $\mathrm{NVH}$, aerospace, structural damping and so on to substitute for magneto-rheological fluids.

In the international academic field, to prepare or improve the stability of the current magneto-rheological fluids, many researchers focus on surface modification of magnetic particles and modified design of the carrier solution. In respect of the surface modification of magnetic particles, Shen et al. ${ }^{2}$ carried on surface modification of micrometer-sized magnetic with zirconia by using a sol-gel method, the coating process dramatically improved oxidation and acid corrosion resistances. Tada et $a l^{3}{ }^{3}$ used fluorocarbon silanes to form the monolayers, which can form a strong chemical bond with the surface of a magnetic particle without degrading its properties. Meanwhile, Park et $a l l^{4,5}$ and Song et al. ${ }^{6,7}$ co-operative group prepared stable magneto-rheological fluids with a core/shell structure and the magnetic particles encapsulated with cross-linked polymer. However, in the condition of high temperature and continuous sheer action, the surface structure of the magnetic particles modified will collapse and lost the stability supported by the special surface modification.

Another method to improve the stability of the magnetorheological material is modified design on the carrier solution. From the point of view that nanoparticles can disperse colloidal molecules, Cao et al. ${ }^{8}$ and Viota et al. ${ }^{9}$ tried to make the nanoparticles with long-range molecular forces to keep in the second energy minimum around the magnetic particles, which form a spatial structure and can improve the stability performance. In order to solve the problem of magnetorheological material with instability and low power transmission property, Gong et al. ${ }^{10,11}$ also implemented research on magneto-rheological fluids and magneto-rheological colloid in China. All these researchers tried to improve the magnetorheological material performance by modify the carrier solution.

Given all that, the research purpose concerning the physical and chemical stability on magneto-rheological fluids is to improve its anti-sedimentation and device reliabilities.

In this study, a new magneto-rheological material, magnetothixotropic gel $^{12}$ is proposed. It accounts for rheological behaviour, correlation between the computational viscosity and rate of shear, correlation between shear stress and rate of shear, field strength and yield stress by using electro-mechanical performance experiments from a macroscopic viewpoint. It hopes to provide basic parameters for magneto-thixotropic gel intelligent devices design. 
TABLE-1

RELATION BETWEEN MAGNETIC INDUCTION AND CURRENT

\begin{tabular}{cccccccccccc}
\hline Current I(A) & 0 & 0.1 & 0.2 & 0.3 & 0.4 & 0.5 & 0.6 & 0.7 & 0.8 & 0.9 & 1.0 \\
Flux B (Gs) & 0 & 149 & 297 & 427 & 574 & 710 & 790 & 870 & 945 & 1025 & 1095 \\
\hline
\end{tabular}

\section{EXPERIMENTAL}

In order to obtain the magneto-thixotropic gel, we prepared it via a chemical and physical mixing process in the laboratory. At first, the mixture of 12-hydroxy stearic acid, stearic acid, sebacic acid had a chemical reaction with the lithium hydroxide and the aqueous solution of calcium hydroxide in the neopentyl polyol fatty acid ester at ca. 160$220^{\circ} \mathrm{C}$ for $4 \mathrm{~h}$. Then the mixture started to flash evaporate and dewater at ordinary temperature and pressure until the water evaporation is complete. After $2 \mathrm{~h}$, it entered into the cooling process, when the temperature decreased to $50{ }^{\circ} \mathrm{C}$ or so, the mixture of magnetic particles, $\beta$-silicon carbide $(\beta$-SiCw) powder, silicone oil and oleic acid were added to the prepared base material with slow stirring. Finally, the magneto-thixotropic gel is prepared.

A schematic diagram for testing the magneto-thixotropic gel's rheological behaviour is presented in Fig. 1. Here, the magneto-thixotropic gel was filled in the gap between the rotor and load rotor. A DC power was employed to excite coil and produce a controllable magnetic field. A sensitive film was adhered to the rotary rod associated with optical tachometer to test the variational sheer speeds. When the rheological behaviour of magneto-thixotropic gel happens under the magnetic field, the magneto-thixotropic gel will become more and more hard with the increasing of field intensity, the rotor torque will be transmitted to load rotor, whose output torque generated through magneto-thixotropic gel rheological behaviour was detected by a torque indicator. The shearing rate of rheological magneto-thixotropic gel can be obtained by a variable-frequency governor (VFG).

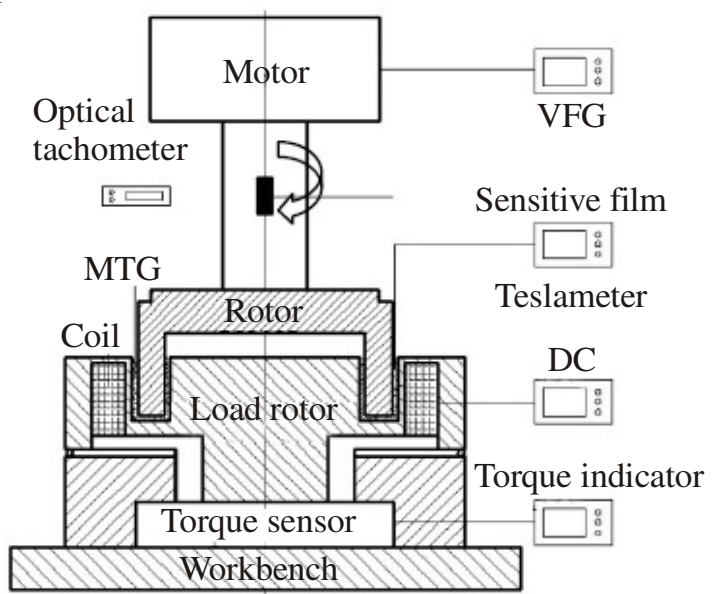

Fig. 1. Schematic diagram of the testing apparatus

Experiment design: To obtain the magnetic induction distribution in the shear zone, it is necessary to pre-test the relationship between magnetic induction and the exciting current. The testing result is shown in Table-1.

The rotation speed series of the rotor varies from 100 to $500 \mathrm{rpm}$ with increment of $50 \mathrm{rpm}$. Hence, the testing results will obtain nine groups of original data included the exciting current, output torque and the corresponding computational viscosity. When the testing begins, the rotation speed was adjusted to the required value every time respectively, then the external magnetic field which matched the exciting current from 0-0.6 A was applied to load on load rotor. And so forth, until the designed rotation speed series was implemented. The data sample format is shown in Table-2.

\begin{tabular}{ccc} 
TABLE-2 \\
\multicolumn{3}{|c}{ CURRENT-OUTPUT TORQUE AND COMPUTATIONAL } \\
VISCOSITY (nr/min) \\
\hline Current I(A) & Torque $\mathrm{M}(\mathrm{Nm})$ & Viscosity $\eta_{\mathrm{Hb}}$ (Pa.s) \\
\hline $\mathrm{I}_{\mathrm{i}}$ & $\mathrm{M}_{\mathrm{ij}}$ & $\eta_{\mathrm{cij}}$ \\
\hline
\end{tabular}

where, $\mathrm{n}(100 \mathrm{rpm} \leq \mathrm{n} \leq 500 \mathrm{rpm})$ presents the different rotate speed; $I_{i}=0,0.1, \ldots, 0.6(i=0,1, \ldots, 6) ; M_{i j}$ and $\eta_{c i j}(j=0,1, \ldots$, 9) signify the output torque of the load rotor and computational viscosity when the magneto-thixotropic gel is exposed to different magnetic field and shear rate.

\section{RESULTS AND DISCUSSION}

Magnetic induction-shear stress properties: In the experimental data from 100-500 rpm, we evenly selected 100 $\mathrm{rpm}, 300 \mathrm{rpm}, 500 \mathrm{rpm}$ as representative data to test the magnetic induction-shear stress properties of the magnetothixotropic gel, the shear stress $\tau_{\mathrm{ij}}$ can be calculated through the equation (1),

$$
\tau_{\mathrm{ij}}=\frac{\mathrm{M}_{\mathrm{ij}} / 2}{2 \pi \mathrm{h}\left(\mathrm{R}_{1}^{2}+\mathrm{R}_{2}^{2}\right)}
$$

where, $M_{i j}$ is output torque with different rotation speed and magnetic induction; $h$ is the valid shear width; $R_{1}$ and $R_{2}$ are respectively the shear radius when the rotor's inner and outer working face works in the U-shaped trough attached to the load rotor. In Fig. 2, on the whole, the curve represents a good linear trend when shear stress changes with the magnetic induction increasing.

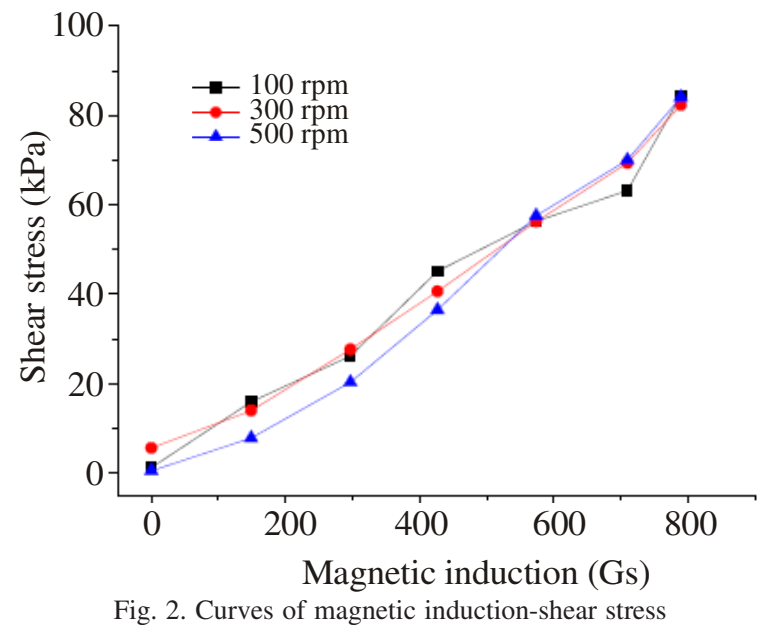

Fig. 2. Curves of magnetic induction-shear stress 
Furthermore, under the different rotation speed, the curves present confluent. It implies that the rotation speeds change has little effect on the shear stress properties, which ensure the magneto-thixotropic gel devices have better controllable performance. This can also be explained through the currentmagnetic induction relation in the Fig. 3, when the exciting current is below 1A, the experimental magnetic induction basically works in linear range, therefore, the shear stress could linearly changes with the magnetic field increasing and at 800 Gs the shear stress can achieve $84 \mathrm{kPa}$, the result is better than $60-75 \mathrm{kPa}$ with magneto-rheological fluids.

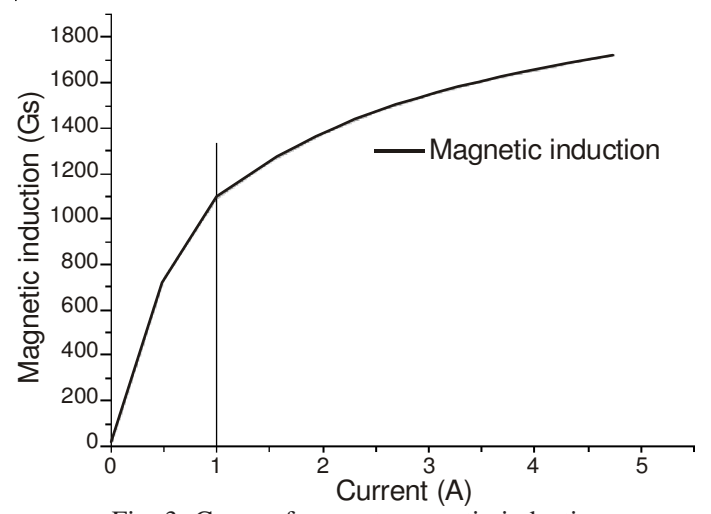

Fig. 3. Curve of current-magnetic induction

Shear strain rate -shear stress properties: Generally, in terms of non-Newtonian fluids like magneto-rheological fluids, affected by shear thinning effect, the shear stress ability presents a decline or instability when shear rotation speed increases. So the shear strain rate-shear stress properties are also a significant item to evaluate the rheological behaviour of the magneto-thixotropic gel. Fig. 4 shows the relation between shear stress and shear strain rate when magneto-thixotropic gel exposed to different magnetic field intensity.

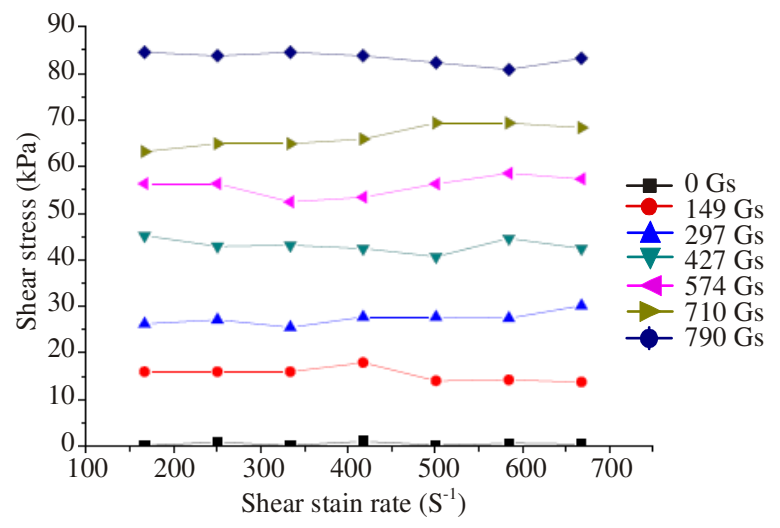

Fig. 4. Relation between shear stress and shear strain rate

When the shear strain rate increases from $167 \mathrm{~S}^{-1}$ to 688 $\mathrm{S}^{-1}$ as well as the magnetic induction from 0Gs (where, 0Gs is only a nominal value, except the first testing data, the magnetic induction has not been zero due to the existence of residual magnetism) to $790 \mathrm{Gs}$, on the whole, the curves at each magnetic field performance a relative stability with little fluctuation. Though the shear strain rate raises, the shear stress basically keeps a linear growth with the increase of magnetic induction.
The stable property of shear stress by changing the strain rate is better than magneto-rheological fluids's and ensure the magneto-thixotropic gel devices qualify for a stable service performance within a wide range of shear speed rate.

Shear strain rate-viscosity properties: The phenomenon that viscosity changes with the shear strain rate, namely also rheological behaviour, is macro performance of magnetothixotropic gel's internal micro-structure under magnetic field and mechanical behaviour. The changing relation not only has something with the internal physical structure, but also subjects to external temperature and the loading force. The magnetothixotropic gel is a non-Newtonian fluid, which decides its viscosity is a variable changing with shear strain rate under certain temperature.

According to the experimental apparatus, the circumferential line speed of load rotor is $v_{c}=\omega_{j} R$, then the radial gradient of $v_{c}$ is:

$$
\frac{d v_{c}}{d R}=R \frac{d \omega_{j}}{d R}+\omega_{j}
$$

where, $\omega$ indicates rotate effect irrelevant to shear effect, therefore, according to the viscosity law, the viscous shear stress of rotor working face $\tau_{\mathrm{ij}}=\eta_{\mathrm{cij}}\left(\mathrm{R} \frac{\mathrm{d} \omega_{\mathrm{j}}}{\mathrm{dR}}\right)$, where $\eta_{\mathrm{cij}}$ namely is the computational viscosity. Then the balancing torque on rotor working face is:

$$
M_{i j}=2 \pi R^{2} h \tau_{i j}=2 \pi R^{3} h \eta_{c i j}\left(\frac{d \omega_{j}}{d R}\right)
$$

Considering $\omega_{j}=2 \pi \eta, \omega_{j}=\int_{R_{1}}^{R_{2}} \frac{M_{i j}}{2 \pi R^{3} h \eta_{c i j}} d R$ then $\eta_{c i j}$ is

$$
\eta_{\mathrm{cij}}=\frac{\mathrm{M}_{\mathrm{ij}}\left(\mathrm{R}_{2}^{2}-\mathrm{R}_{1}^{2}\right)}{8 \pi^{2} h R_{1}^{2} R_{2}^{2} \mathrm{n}}
$$

where, $\mathrm{M}_{\mathrm{ij}}$ is the output torque displayed on the torque indicator and $\mathrm{n}$ is the rotation speed of the rotor. In the experiment, when the magnetic induction increases from zero to $790 \mathrm{Gs}$, under the different shear strain rate, the magneto-thixotropic gel represents different viscosity changing trend that is shown in Fig. 5.

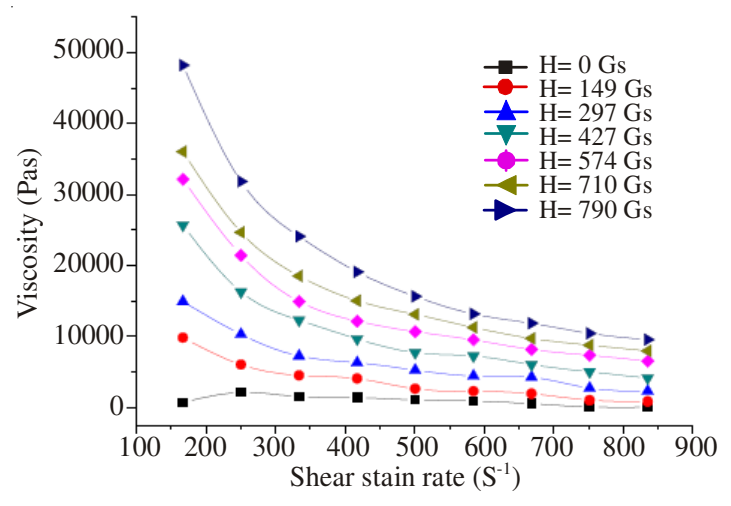

Fig. 5. Shear strain rate-viscosity changing with magnetic field

Fig. 5 shows when the rheological behaviour takes place under a lower shear strain rate and the certain magnetic field, the magneto-thixotropic gel can obtain a more large viscosity, 
when the magnetic induction increases to $790 \mathrm{Gs}$ with strain rate being $168 \mathrm{~s}^{-1}(\mathrm{n}=100 \mathrm{rpm})$, the computational viscosity achieves $4.82 \times 10^{4} \mathrm{~Pa} \cdot \mathrm{s}$. The result signifies that under the low shear strain rate and the strong magnetic field, the magneto-thixotropic gel could acquire greater capacity of resistance to deformation and shearing. Meanwhile, under the same shear strain rate, basically the viscosity linearly increases with the increasing of magnetic induction. The change trend matches the changing relation between shear stress asnd shear strain rate. This also explained why the magneto-thixotropic gel has a potentially higher shear yield stress than magnetorheological fluids.

\section{Conclusion}

An experimental investigation and theoretical analysis were conducted to characterize the rheological behaviour of magneto-thixotropic gel acting on changing magnetic field and the shear strain rate. Three groups of interactive parameters were selected for this study namely, magnetic induction-shear stress properties, shear strain rate -shear stress properties and shear strain rate-viscosity properties. The main conclusions are summarized as follows:

The magneto-thixotropic gel has an outstanding linear magnetization property. The shear stress could linearly changes with the magnetic field increasing and at $800 \mathrm{Gs}$ the shear stress can achieve $84 \mathrm{kPa}$, the result is much better than 60-75 $\mathrm{kPa}$ magneto-rheological fluids. The feature enhances the magneto-thixotropic gel devices' power transmission ability and control accuracy.

When the shear strain rate increases from $167 \mathrm{~S}^{-1}$ to 688 $\mathrm{S}^{-1}$ as well as the magnetic induction from $0 \mathrm{Gs}$ to $790 \mathrm{Gs}$, the shear stress performances a relative stability and basically keeps a linear growth with the increase of magnetic induction. Compared with magneto-rheological fluids, the performance ensures the magneto-thixotropic gel devices qualify for a stable service performance within a wide range of shear speed rate.

Under the low shear strain rate and the strong magnetic field, the magneto-thixotropic gel could acquire greater capacity of resistance to deformation and shearing than current magneto-rheological material. The viscosity of the magnetothixotropic gel will rise with the decrease of the shear strain rate and with increasing of magnetic induction. The computational viscosity achieves $4.82 \times 10^{4} \mathrm{~Pa} \cdot \mathrm{s}$ when is at point magnetic induction $790 \mathrm{Gs}$ with strain rate being $168 \mathrm{~s}^{-1}$.

\section{ACKNOWLEDGEMENTS}

This research was partly supported by the Director Prospective Foundation (Y10910QF06) funded by Chinese Academy of Sciences and China Postdoctoral Science Foundation (No. 2011M500145). It was also supported by the Ningbo Natural Science Foundation (2011A610136) sponsored by Ningbo Scientific and Technological Commission. Thanks are also due to Prof. Changrong Liao of Chongqing University for his help during the experiment.

\section{REFERENCES}

1. H.Z. Lv, X.C. Liang and J.X. Wang, Mater. Sci. Technol., 19, 139 (2011).

2. R. Shen, S.N. Shafrir, C.L. Miao, M.M. Wang, J.C. Lambropoulos, S.D. Jacobs and H. Yang, J. Colloid. Interf. Sci., 342, 49 (2010).

3. T. Tada, S. Onishi, Y. Ohkubo and K. Ogawa, Thin Solid Films, 517, 4360 (2009).

4. B.J. Park, M.S. Kim and H.J. Choi, Mater Lett., 63, 2178 (2009).

5. B.J. Park, J.L. You, H.J. Choi, IEEE Trans. Magnet., 45, 2460 (2009).

6. K.H. Song, B.J. Park and H.J. Choi, IEEE Trans. Magnet., 45, 4045 (2009).

7. K.H. Song, B.J. Park and H.J. Choi, 3rd International Nanoelectronics Conference, January 3-8; Hongkong (2010).

8. L.F. Cao, H. Park, G. Dodbiba and T. Fujita, Int. J. Mod. Phys. B, 24, 1227 (2010)

9. J.L. Viota, F. González-Caballero, J.D.G. Durán and A.V. Delgado, J. Coll. Interf. Sci., 309, 135 (2007).

10. W. Zhang, X.L. Gong, S.H. Xuan and Y.G. Xu, Ind. Eng. Chem. Res., 49, 12471 (2010)

11. B. Wei, X.L Gong, W.Q. Jiang, L.J. Qin and Y.C. Fan, J. Appl. Polym. Sci., 118, 2765 (2010).

12. H.Z. Lv and S. Xiao, China Patent ZL200910191876.8, 24 Jun (2011). 